\title{
Nondestructive Damage Detection of a Magnetostrictive Composite Structure ${ }^{\dagger}$
}

\author{
Asha Hall 1,*, Michael Coatney ${ }^{1,}$ Natasha Bradley ${ }^{1}$, Jin Hyeong Yoo ${ }^{2}$, Nicholas Jones ${ }^{2}$, \\ Brandon Williams ${ }^{3}$ and Oliver Myers ${ }^{3}$ \\ 1 US Army Research Laboratory, 6340 Rodman Rd., Aberdeen Proving Ground, MD 21005, USA; \\ michael.d.coatney.civ@mail.mil (M.C.); natasha.c.epps-bradley.civ@mail.mil (N.B.) \\ 2 US Naval Surface Warfare Center (NSWC), 9500 MacArthur Blvd, Bethesda, MD 20817, USA; \\ jinhyeong.yoo@navy.mil (J.H.Y.); nicholas.j.jones1@navy.mil (N.J.) \\ 3 Clemson University, 244 Fluor Daniel Building, Clemson, SC 29634, USA; bew3@g.clemson.edu (B.W.); \\ omyers@clemson.edu (O.M.) \\ * Correspondence: asha.j.hall.civ@mail.mil; Tel.: +1-410-278-2384 \\ + Presented at the 18th International Conference on Experimental Mechanics, Brussels, Belgium, 1-5 July \\ 2018.
}

Published: 28 May 2018

\begin{abstract}
The integrity of composite structures gradually degrades due to the onset of damage such as matrix cracking, fiber/matrix debonding, and delamination. Over the last two decades, great strides have been made in structural health monitoring (SHM) community using various sensing techniques such as acoustic emission, eddy current, strain gages, etc., to diagnose damage in aerospace, mechanical and civil infrastructures. Embedded sensing offers the prospects of providing for real-time, in-service monitoring of damage were weight savings is a major factor in Aerospace Industry. In this present work, magnetostrictive particles such as Terfenol-D were embedded in a composite structure, along with multiple SHM techniques, to capture the damage in an IM7-carbon fiber reinforced polymer composite system undergoing fatigue loading. As the internal stress state increases, the change in the magnetization flux intensity was captured using a non-contact magnetic field sensor. A damage diagnosis system was established along with an acoustic emissions technique to further validate the damage captured by the embedded system. The goal of this project is to identify the change in the mechanical and magnetic property within a composite material during the evolution of damage. Several characterization techniques were used to determine interfacial fiber-matrix interactions which will provide for a more comprehensive understanding of the composite interfaces.
\end{abstract}

Keywords: structural health monitoring; magnetostrictive materials; embedded nanocomposites

\section{Introduction}

The integrity of composite structures gradually degrades due to the onset of damage such as matrix cracking, fiber/matrix debonding, and delamination. Over the last two decades, great strides have been made in structural health monitoring (SHM) community using various sensing techniques such as acoustic emission, eddy current, strain gages, etc., to diagnose damage in aerospace, mechanical and civil infrastructures. While various conventional non-destructive evaluation (NDE) techniques are used to detect, monitor, and visualize damage of composite structures under fatigue loading, they all have a common limitation in that continuous damage assessment cannot be made in real-time while in service [1-4]. Our current research examines the possibilities of embedding nanomaterials into composite laminates as a minimally invasive, non-contacting, nondestructive evaluation and sensing technique. The embedded sensing composite effectively becomes a smart 
structure with the capability of self-sensing damage by evaluating the integrity of the material in realtime.

Ferromagnetics possess unpaired electronic spins that line up parallel with each other in a region called a domain. The domains are randomly oriented when the ferromagnetic material is not under an external magnetic field. Once an external magnetic field is applied the electronic spins line with each other and become magnetized.Magnetostriction is a property of ferromagnetic materials such that when an external magnetic field is applied the domains align to the external field causing their shape or dimensions to change during the process of magnetization. For example, TerFenol-D, ( $\mathrm{Tb}_{0.3 \mathrm{Dy}} \mathrm{Dy}_{\mathrm{.}} \mathrm{Fe}_{1.92}$ ) strains to about 2000 microstrain in a field of $2 \mathrm{kOe}$ when under a mechanical-bias [5-7]. Due to their high magnetostrictive coefficients TerFenol-D serves as an excellent candidate for in-situ sensing of damage in various kinds of polymer composites under different mechanical loading situations $[8,9]$.

Ferromagnetic materials have proven useful as strain transducers in harsh-environment applications. Magnetostrictive materials such as Galfenol (Fe-Ga alloy), Alfenol (Fe-Al alloy), and

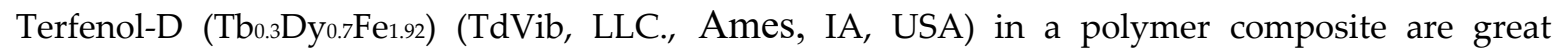
candidates for embedded damage assessment of composite structures. In this study, magnetostrictive particles such as Terfenol-D were embedded in a composite structure, along with acoustic emissions technique, to validate the damage in a composite system undergoing qausi static and fatigue loading. As the applied load and fatigue cycles increased, the change in the magnetization flux density was captured using a non-contact magnetic field sensor. It was confirmed through numerous tests that a change in the magnetic properties of the composite served as an indicator of early stage damage detection.

\section{Experimental}

A 12 in. wide unidirectional carbon fiber reinforced polymer (CFRP) prepreg (Hexcel IM7/8552, 190gsm Prepreg) was used to fabricate the panels. $20 \mathrm{wt} \%$ of Terfenol-D particles were embedded into gage length of each layer of carbon fiber reinforced polymer unidirectional pre-pregs with a total of 8 plies. The particle size of the powders ranged from 1-200 microns and were distributed evenly within the gage length of the ply. The prepreg was chosen for being well known as a high performance material in the aerospace industry, also allowing for easy embedding of the magnetostrictive particles due to its sticky nature. Panels were produced using eight plies of the prepreg arranged with the $(0 / 90)$ orientation. The laminate was then vacuum bagged with the construction. The temperature of the vacuum bagged panel was ramped from RT to $225^{\circ} \mathrm{F}$ at $5{ }^{\circ} \mathrm{F} / \mathrm{min}$ and then held for $60 \mathrm{~min}$ at approximately $15 \mathrm{psi}$ absolute pressure (ambient). Pressure was then increased to $100 \mathrm{psi}$, and the temperature was ramped up to $300^{\circ} \mathrm{F}$ at $5^{\circ} \mathrm{F} / \mathrm{min}$. As the temperature surpassed $240^{\circ} \mathrm{F}$, a 120-min soak clock was started, and the vacuum, vented. At the end of the 120min soak period, temperature was ramped down to $70^{\circ} \mathrm{F}$ at $5{ }^{\circ} \mathrm{F} / \mathrm{min}$ and then the pressure was released. The dimensions of the specimen were water jet cut into 1in. width and $10 \mathrm{in}$. in length, according to ASTM standard D3039 for polymer matrix composite materials. Tabs were applied to the ends of the samples using G-10 fiberglass laminate to aid in distributing the loads to the center of the specimens. Baseline samples (non-embedded Terfenol-D panels) were prepared with exactly the same dimension and thickness to serve as a standard. 


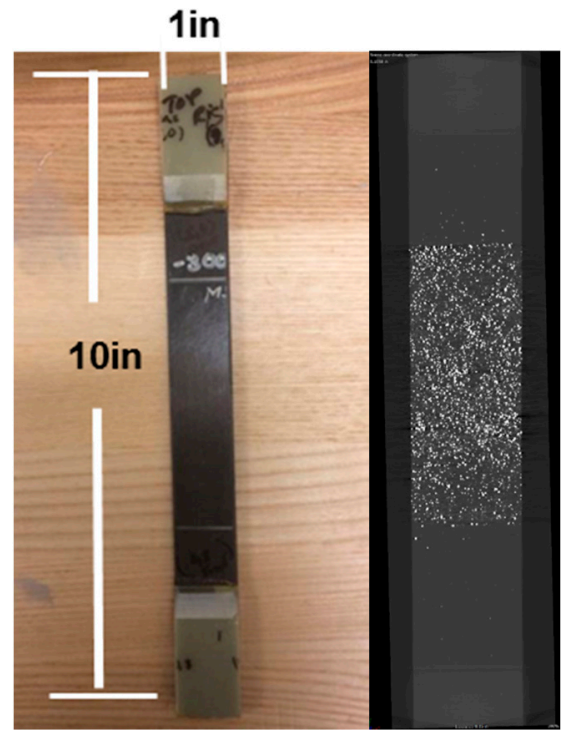

Figure 1. X-ray Micro CT image of the IM7/8552 CFRP with Terfenol-D particles.

A Zeiss X-ray Micro CT revealed that the Terfenol-D particulates were uniformly distributed within the gage length of the samples as shown in Figure 1. This characterization tool allowed us to visualize the structural detail of the addition of the magnetostrictive particles at every layer. Figure 2 depicts the CFRP sample inserted into a Mechanical Testing System (MTS) machine for preparation of a quasi-static tension-tension test. The driving coil/pick-up coil sensor is mounted about $2 \mathrm{~mm}$ from the surface of the sample. The driving coil sends a magnetic field to the specimen while the pickup coil receives the change in voltage signal/magnetic flux density.

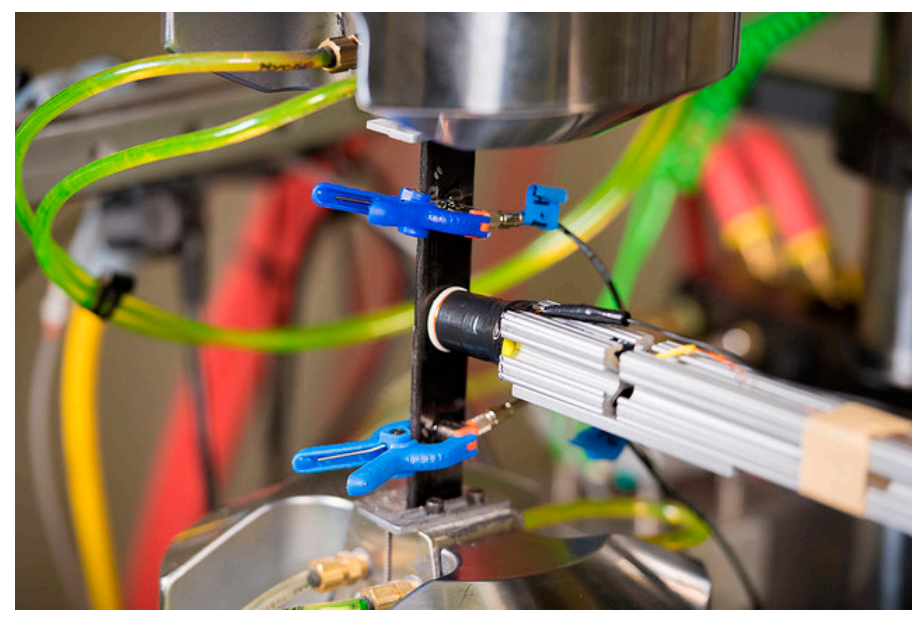

Figure 2. Magnetostrictive carbon fiber reinforced polymer. Quasi static Tension-Tension Test.

\section{Results and Discussion}

Figure 3 depicts a stress - strain curve of a magnetostrictive CFRP sample. The elastic modulus of the Non Magnetostrictive CFRP sample was $120 \mathrm{GPa}$, while the Elastic Modulus of the magnetostrictive sample is $115 \mathrm{GPa}$, which is a $7 \%$ decrease in the failure stress. This indicates a slight decrease in strength for the Terfenol-D composite samples. The slope of the linear elastic region of the baseline CFRP yielded a yield strength of $12.8 \mathrm{MPa}$. The specimen fractured in the middle of the gage with longitudinal splitting and tearing. The magnetostrictive specimens were also tested to failure in order to compare the yield strength of the pristine versus magnetostrictive.

The results from experimental tensile testing of unidirectional carbon fiber reinforced polymer composite specimens with and without magnetostrictive particles showed that the particle layer was slightly intrusive on the quasi-static tension properties of the beam. Based on the number of samples 
tested, the amount of change seen in both the tensile strength and the modulus was statistically negligible. Future tests will be conducted incorporating acoustic emission to further validate the correlation between the changes in magnetic flux intensity to the increase in microcrack density.

Initial tests were measured to find the ultimate tensile strength (UTS) of the $20 \mathrm{wt} \%$ specimens. Once the UTS was known, the next set of samples were loaded at $10 \%$ intervals, up to $70 \%$ of the UTS. At each load interval, the load was held while a signal was recorded for approximately 3 to 4 sec at a $5000 \mathrm{~Hz}$ sampling rate. Figure 4 depicts the peak voltage of the sample as each load intervals were applied. At 50\% applied load there is a decrease in voltage RMS (i.e. magnetic flux density) of the specimen as well as a slight frequency shift. There is a suspicion of micro cracking occurring at the $50 \%$ UTS applied load which would have shifted resonance frequency. On the contrary, as the UTS percentage increases to the $60 \%$ and $70 \%$ UTS the voltage RMS returns to the initial voltage RMS as recorded earlier in the loading regime. The presence of internal stresses in a structure could influence the domain boundary movement of magnetostrictive particles in the structure which can be detected by an external magnetic sensor thereby revealing the early stage of the initiation of damage.

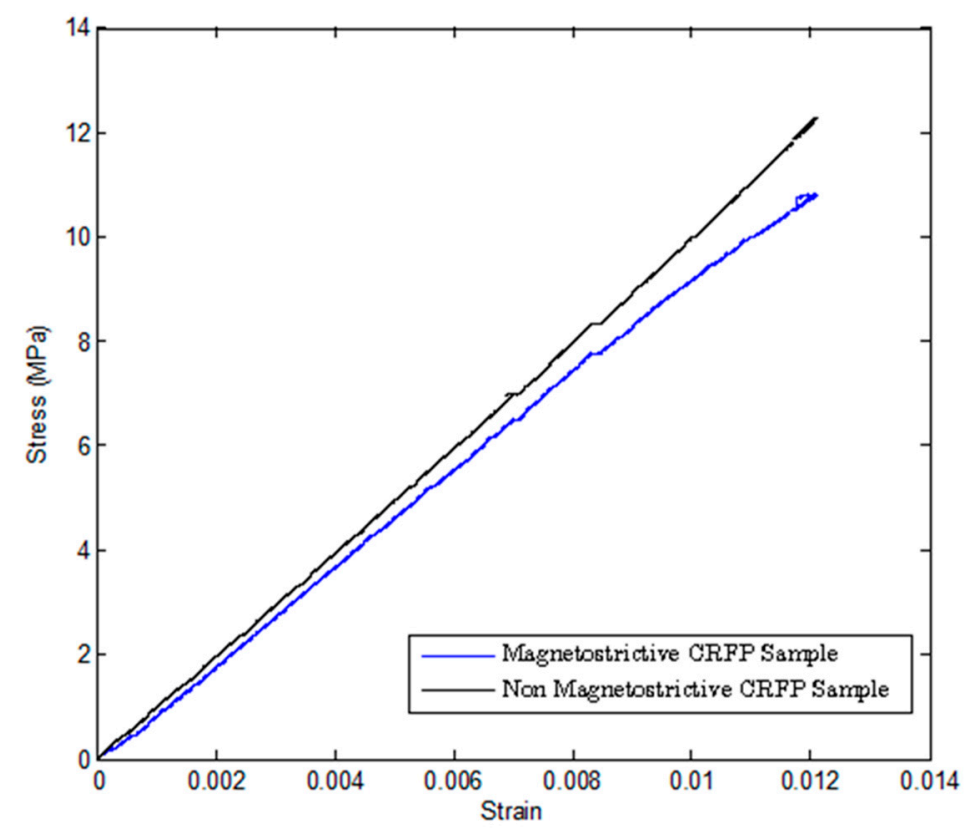

Figure 3. Stress-Strain Curve. Elastic modulus of the Non Magnetostrictive and Magnetostrictive CFRP sample.

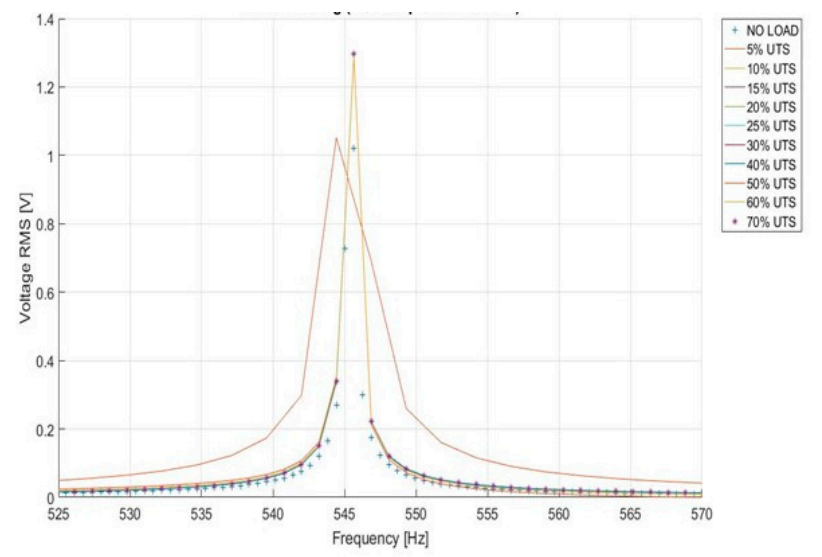

Figure 4. Voltage RMS as a function of frequency. A change in voltage RMS from $50 \%$ of the ultimate tensile strength (UTS) to $60 \%$ UTS indicated degradation of the specimen. 


\section{Conclusions}

In this study, magnetostrictive Terfenol-D powders were embedded in Terfenol-D composite specimens. This technique was performed to examine the changes in magnetostriction from tensile fatigue loading. The results from experimental tensile testing of $(0 / 90)$ carbon fiber reinforced polymer composite specimens with and without magnetostrictive particles showed that the particle layer was minimally intrusive on the quasi-static tension properties of the beam. Based on the number of samples tested, the amount of change seen in both the tensile strength and the modulus was statistically negligible. Future tests will be conducted incorporating acoustic emission to further validate the correlation between the changes in magnetic flux intensity to the increase in microcrack density.

Author Contributions: A.H. and J.H.Y. conceived and designed the experiments; M.C. and N.B. performed the experiments; B.W., and O.M. analyzed the data; J.H.Y. contributed to the characterization and analysis tools; A.H. wrote the paper.

Conflicts of Interest: The authors declare no conflict of interest.

\section{References}

1. Wang, S.; Shui, X.; Fu, X.; Chung, D.D.L. Early Fatigue Damage in Carbon-Fibre composites Observed by Electric Resistance Measurements. J. Mater. Sci. 1998, 33, 3875-3884.

2. Hall, A.J.; Brennan, R.E.; Ghoshal, A.; Liu, K.C.; Coatney, M.; Haynes, R.; Bradley, N.; Weiss, V.; Tzeng, J., Damage Precursor Investigation of Fiber-Reinforced Composite Materials Under Fatigue Loads; US Army Research Laboratory Technical Report/ARL-TR-6622; Aberdeen Proving Ground, MD, USA, September 2013.

3. ASTM Standard D3039, 2007. Standard Test Method for Tensile Properties of Polymer Matrix Composite Materials; ASTM International: West Conshohocken, PA, USA, 2007; doi:10.1520/D3039_D3039M-08,

4. Ramsteiner, F.; Ambrust, T. Fatigue Crack Growth in Polymer. Polym. Test. 2001, 20, 321-327.

5. Mudivarthi, C.; Datta, S.; Atulaimha, J.; Flatau, A.B. A bidirectionally coupled magnetoelastic model and its validation using a Galfenol unimorph sensor. Smart Mater. Strut. 2008, 17, 035005.

6. Calkins, F.T.; Flatau, A.B.; Dapino, M.J. Overview of Magnetostrictive Sensor Technology. J. Intell. Mater. Syst. Struct. 2007, 18, 1057-1066.

7. Purekar, A.; Ragunath, G.; Flatau, A.B.; Yoo, J.-H. Development of Galfenol Based Non-Contact Torque Sensor. In Proceeding of the 53rd AIAA/ASME/ASCE/AHS/ASC Structures, Structural Dynamics and Materials Conference, Honolulu, HI, USA, 23-26 April 2012.

8. Clark, A.E.; Abbundi, R.; Gillmor, W.R. Magnetization and magnetic anisotropy of TbFe2, DyFe2, Tb0.27Dy0.73Fe2 and TmFe2. IEEE Trans. Magn. 1978, 14, 542-544.

9. Or, S.W.; Nersessian, N.; Carman, G.P. Dynamic Magnetomechanical Behavior of Terfenol-D/Epoxy 1-3 Particulate Composites. IEEE Trans. Magn. 2004, 40, 71-77.

(C) 2018 by the authors. Licensee MDPI, Basel, Switzerland. This article is an open access article distributed under the terms and conditions of the Creative Commons Attribution (CC BY) license (http://creativecommons.org/licenses/by/4.0/). 